- Recognise when tooth surface loss is pathological

- Determine the aetiology of tooth surface loss

- Help the patient to decide whether treatment is indicated

- Devise an orderly framework for the design of treatment plans

- Provide a rationale for treatment of difficult cases

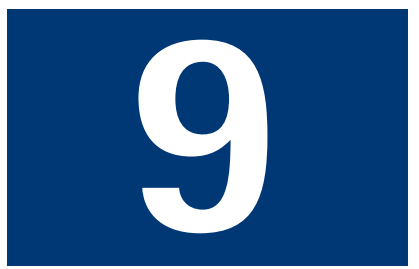

\title{
Management of tooth surface loss
}

\author{
S. J. Davies ${ }^{1}$ R. J. M. Gray ${ }^{2}$ and A. J. E. Qualtrough ${ }^{3}$
}

This part of the series is devoted to tooth surface loss (TSL) not caused by caries or trauma. The management of this form of generalised TSL is included in this series because knowledge of occlusion is needed for both the diagnosis and, when indicated, treatment. There are, however, many other factors involved in the management of generalised TSL other than those associated with 'occlusion'. These will also be discussed.

\section{Tooth Surface Loss physiological? pathological?}

${ }^{*}$ GDP, 73 Buxton Rd, High Lane, Stockport SK6 8DR; P/T Lecturer in Dental Practice, University Dental Hospital o Manchester, Higher Cambridge St., Manchester M15 6FH: ${ }^{2}$ Honorary Fellow, University Dental Hospital of Manchester Higher Cambridge St., Manchester M15 6FH ${ }^{3}$ Senior Lecturer and Honorary Consultant in Restorative Dentistry, University Dental Hospital of Manchester, Higher Cambridge St., Manchester M15 6FH

${ }^{*}$ Correspondence to : Stephen Davies,

73 Buxton Rd, High Lane, Stockport SK6 8DR

Email:stephen.j.davies@man.ac.uk

\section{Refereed Paper}

(c) British Dental Journal 2002; 192:

11-23
Is the tooth surface loss physiological or pathological?

Tooth surface loss may be purely physiological (Fig. 1) and occurs as a natural consequence of ageing. ${ }^{1}$ Several factors, however, including erosion, abrasion and attrition can render tooth surface loss pathological (Fig. 2). As a result of this, symptoms may develop and treatment may be indicated. Although this chapter will deal with only pathological tooth surface loss, it is important to be able to recognise when tooth surface loss is purely physiological; it cannot be assumed that all tooth surface loss is pathological.

\section{CRITERIA OF PHYSIOLOGICAL TOOTH SURFACE LOSS}

There appears to be no consensus as to what constitutes physiological tooth surface loss. It would be of assistance to practising dentists, if such criteria could be established. In the absence of accepted criteria of physiological tooth surface loss, those of pathological tooth surface loss are presented in Figure 2.

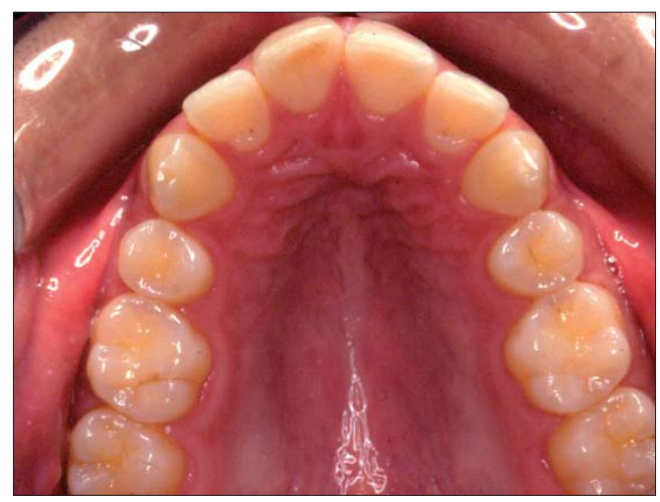

Fig. 1 Purely physiological tooth surface loss

\section{CLASSIFICATION OF TOOTH SURFACE LOSS}

There are usually considered to be three reasons for non carious tooth surface loss. Abfractions should also be considered to be a cause of noncarious TSL.

\section{Erosion}

Erosion is a chemical process in which the tooth surface is removed in the absence of plaque. ${ }^{2}$ Erosive factors may be either intrinsic or extrinsic. Extrinsic sources include drinks such as fresh fruit juices, carbonated drinks, cordials and alcoholic beverages; and some foods and industrial processes. Intrinsic sources include gastrooesophageal reflux and eating disorders, amongst others.

\section{Abrasion}

External agents which have an abrasive effect on the teeth include toothbrush bristles and dietary factors.

\section{Attrition}

Attrition is a process in which tooth tissue is

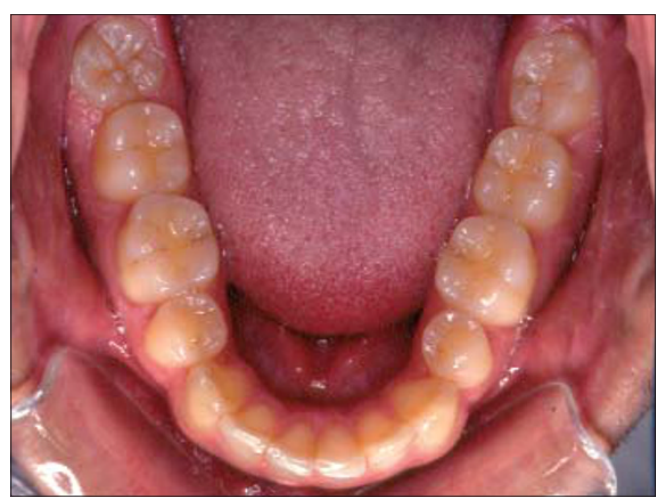


Fig. 2 Pathological tooth surface loss may result in one or more of the following

\section{Fig. 3 Abfraction}

\section{Terminology:}

Function
a normal movement

Parafunction
a normal movement
at an abnormal
frequency

Dysfunction an abnormal movement
- Change in appearance of teeth

- Pain and/or sensitivity

- Loss in occlusal vertical dimension

- Loss in posterior occlusal stability resulting in - Increased tooth wear

- Mechanical failure of teeth or restorations

- Hypermobility and drifting
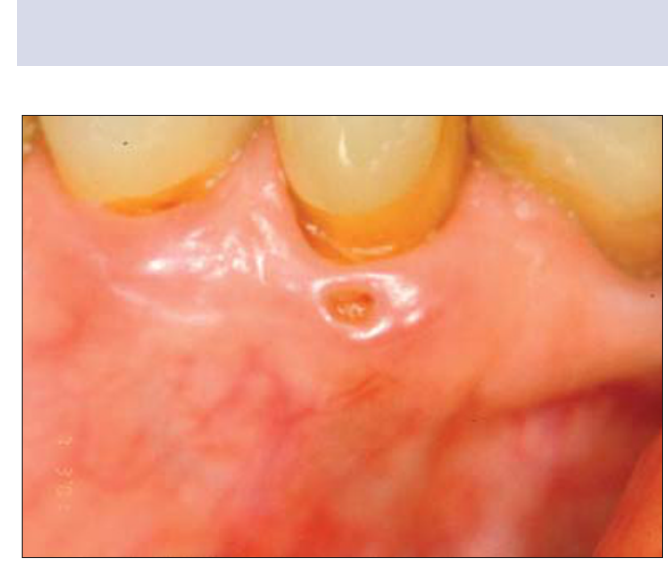

removed as a result of opposing tooth surfaces contacting during function or parafunction. Such direct contact occurs at proximal areas, on supporting cusps and on guiding surfaces during empty grinding movements.

\section{Abfractions (stress lesions)}

It has been suggested that the stress lesion or abfraction is a consequence of eccentric forces on the natural dentition. . $^{3,4}$ The theory propounds tooth fatigue, flexure and deformation via biomechanical loading of the tooth structure, primarily at the cervical regions. Cusp flexure causes stress at the cervical fulcrum and results in loss of the overlying tooth structure. The lesion is typically wedge shaped with sharp line angles, but occlusal abfractions may present as circular invaginations. The magnitude of tooth tissue loss depends on the size, duration, direction, frequency and location of the forces. It should be remembered that abfractive lesions are caused by flexure and fatigue of susceptible teeth at sites that are usually distant from the point of loading. Other factors, such as erosion and abrasion may play a significant role in tooth tissue loss, but the initial force is the biomechanical loading.

Figure 3 shows a cervical cavity which is significantly subgingival and interestingly has caused a fenestration of the overlying attached gingiva. It is highly likely, because of the protection of the overlying soft tissue, that this lesion is an abfraction without any abrasive or erosive component.

\section{BRUXISM}

\section{What is 'Bruxism'?}

Bruxism is an important factor related to tooth surface loss. It is defined as the grinding of teeth during non functional movements of the masticatory system: it is a mandibular parafunction.
The wear is usually uniform when opposing teeth are affected. If bruxism is severe, either marked wear of occlusal surfaces will occur or, in cases of compromised periodontal support, tooth mobility may result. Bruxism can also be associated with muscle spasm, fractured teeth and restorations. ${ }^{5}$

\section{What are the signs of active bruxism?}

Tooth surface loss or tooth wear cannot be taken as a sign that the patient is an active bruxist. Even if the cause of the TSL was bruxism the patient may no longer be bruxing. The signs of active bruxism are tongue scalloping and cheek ridging. ${ }^{6}$

\section{What causes bruxism?}

Two aetiological models have been proposed:

(i) The structural model, which is based upon the role played by malocclusion or by an alteration in maxillo-mandibular relationship.

(ii) The functional model, which highlights the effects of physiological stress as a predominant cause.

There are, however, no reliable predictive indicators to suggest a simple relationship between the causes and effects of bruxism. It was once thought that 'Type A personalities' were more susceptible to stress related bruxism, but current teaching discounts this classification as a gross oversimplification and it is no longer generally used.

\section{Physiological tooth wear}

As proximal contacts wear in a normal dentition, there is a compensatory occlusal adjustment. This may happen naturally if the diet is abrasive ie this is as a result of function, not parafunction, and so should be not be considered pathological tooth surface loss caused by bruxism.

\section{How can bruxism be treated?}

It has been found that the signs and symptoms of bruxism often disappear when occlusal therapy is aimed at the provision of an ideal occlusion: that is the careful elimination of interferences in the static and dynamic occlusion and the maximal distribution of occlusal load. Occlusal therapy should only be carried out after successful stabilisation splint usage, and careful 'mock' equilibration on accurately mounted study models. The need for a period of splint therapy is reinforced by the fact that strong clenching has the effect of compressing the periodontal ligament and rebound may take more than half an hour. This is of particular significance when the occlusion is either being assessed or equilibrated.

Furthermore, if the aim is to discourage a bruxist habit, this may be achieved by the patient's intermittent use of an occlusal splint; a stabilisation splint or a localised occlusal 


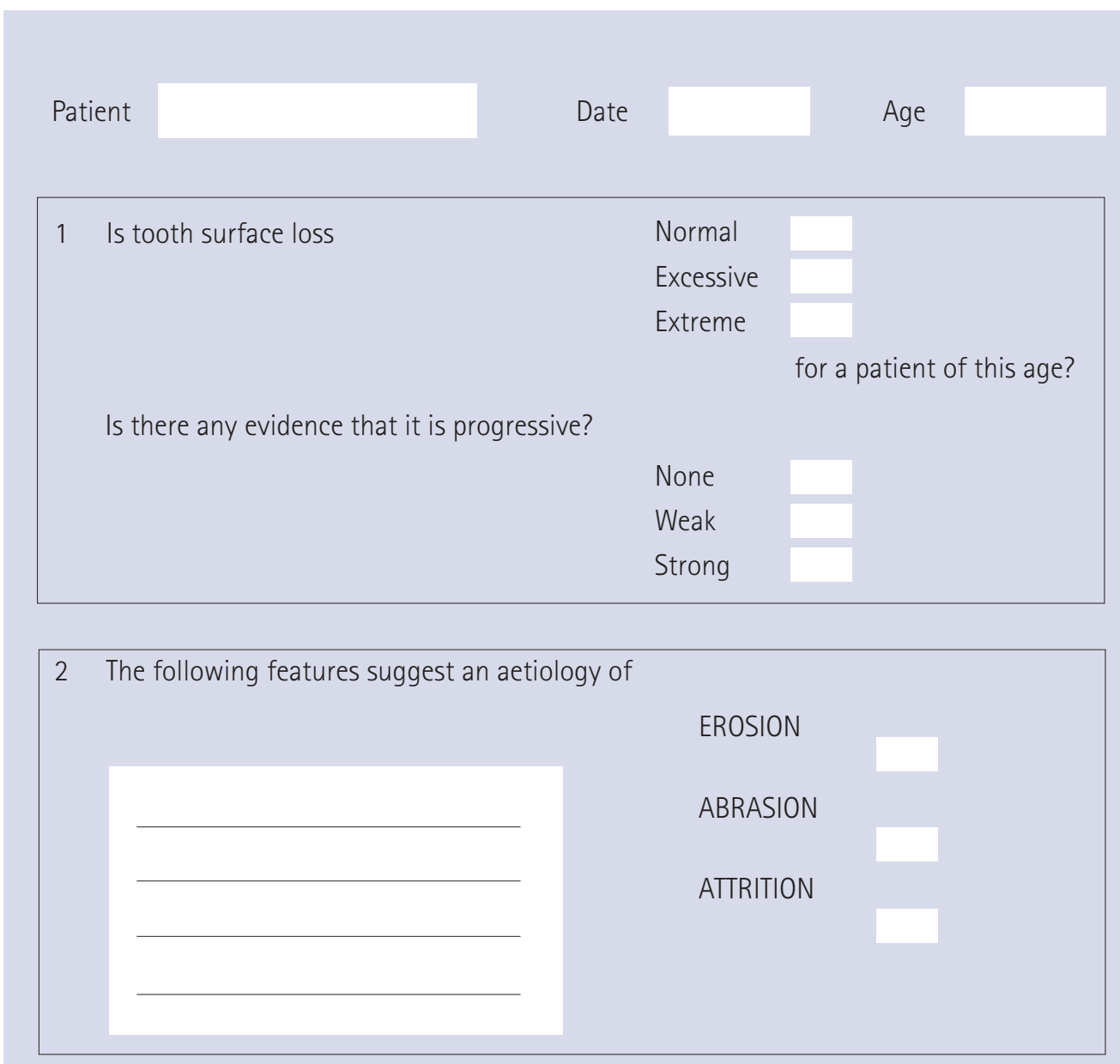

3 Known dietary habit?

Known gastric reflux?

Known parafunction?

Other [specify]?

$4 \quad$ Has dento-alveolar compensation taken place?

5 QUALTY OF LIFE ISSUES

Patient is worried about:

Appearance

Tooth sensitivity

Tooth or restoration fracture

Soft tissue comfort

Loosening teeth

6 RECORDS taken and TESTS made

Centric Relation jaw registration

Models

Photographs

Tooth measurements taken

Ethyl chloride 
interference splint, or in some cases a soft bite guard.

\section{TECHNIQUE}

\section{Examination}

The patient for whom a diagnosis of pathological tooth surface loss is suspected, should be given a particular examination (Fig. 4). This is in addition to the normal examination of the articulatory system .

This examination is designed to:

- Aid in distinguishing between physiological and pathological TSL

- Reveal any features which may indicate the aetiology

- Indicate whether or not treatment should be carried out

- Highlight any potential difficulties anticipated in treatment.

Is the tooth surface loss pathological ?

This question gives rise to Box 1 of the examination sheet (Fig. 4)

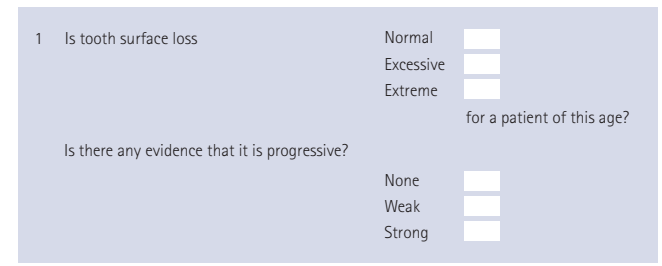

The features of pathological tooth surface loss: Pathological tooth surface loss may result in a change in the appearance of teeth, considered to be excessive with respect to the age of the patient (Fig. 5). ${ }^{7}$

There may be:

- Sensitivity to thermal stimuli

- A loss in vertical height

- A history of frequent fracture of teeth or restorations

- Hypermobility and drifting.

\section{Are there any features of tooth} surface loss which suggest a particular aetiology?

This question gives rise to Boxes 2, 3 of the examination sheet (Fig. 4).
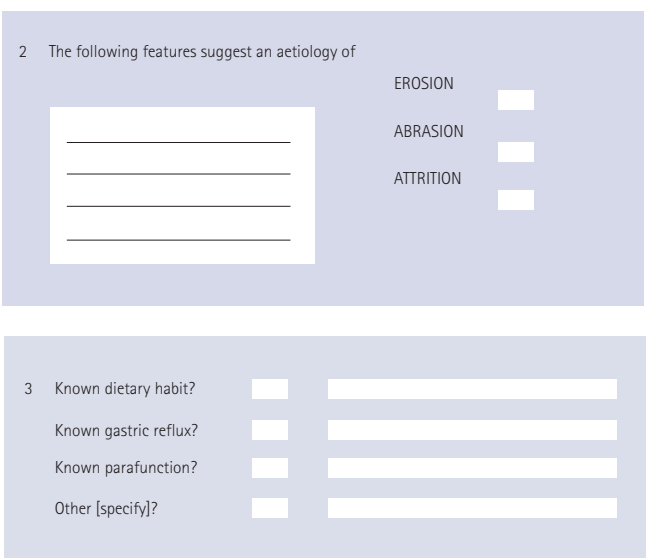
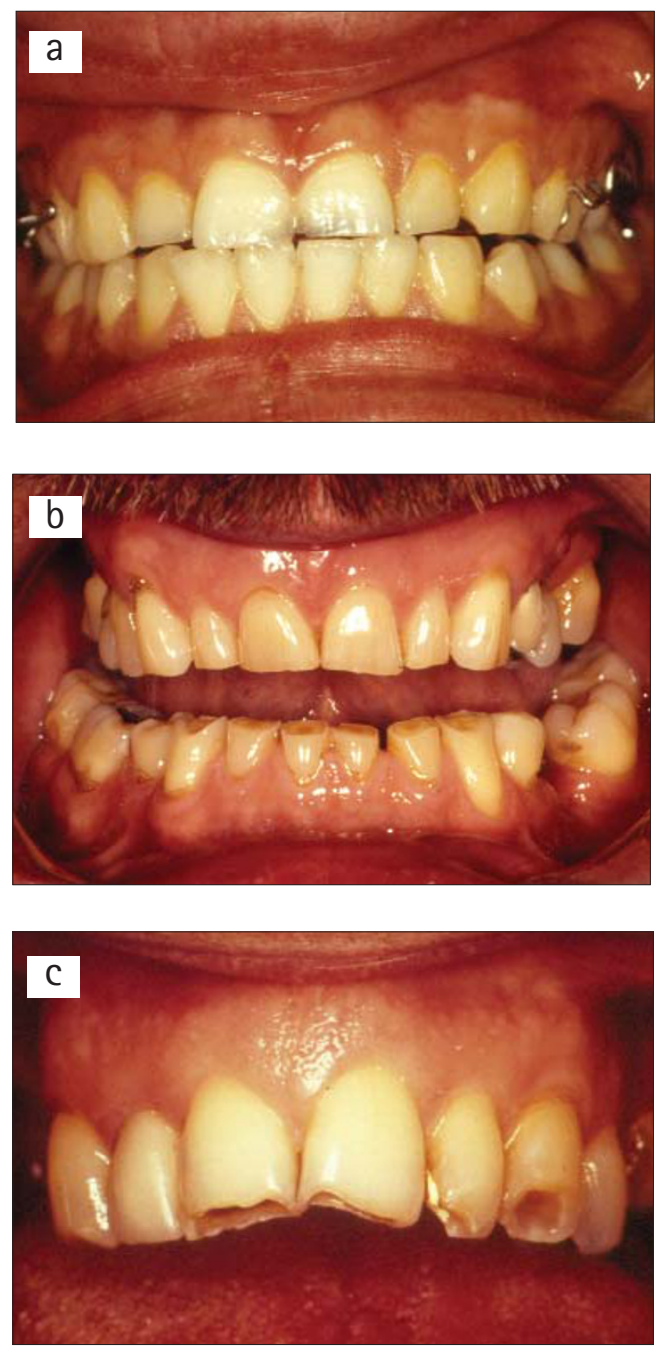

Fig. 5: Fig. 5a shows a young patient (18 years), who needs treatment, whereas in Fig. $5 \mathrm{~b}$ a similar amount of TSL in an older (75 years) patient does not necessarily warrant intervention. The patient in Fig. 5c is $\mathbf{4 5}$ years old and his request for treatment is justified

The features of different types of tooth surface loss

Despite the multi-factorial aetiology of tooth surface loss, certain clinical features may suggest a major contributory factor. Flattening of cusps or incisal edges and localised facets on occlusal or palatal surfaces would indicate a primarily attritional aetiology. Traditionally, cervical lesions caused purely by abrasion have sharply defined margins and a smooth, hard surface. The lesion may become more rounded and shallow if there is an element of erosion present. Once dentine is exposed, the clinical appearance is determined by the relative contribution of the aetiological factors. If wear is primarily attritional, then dentine tends to wear at the same rate as the surrounding enamel. Erosive lesions cause 'cupping' to form in the dentine. When erosion affects the palatal surfaces of upper maxillary teeth, there is often a central area of exposed dentine surrounded by a border of unaffected enamel. ${ }^{8}$ 


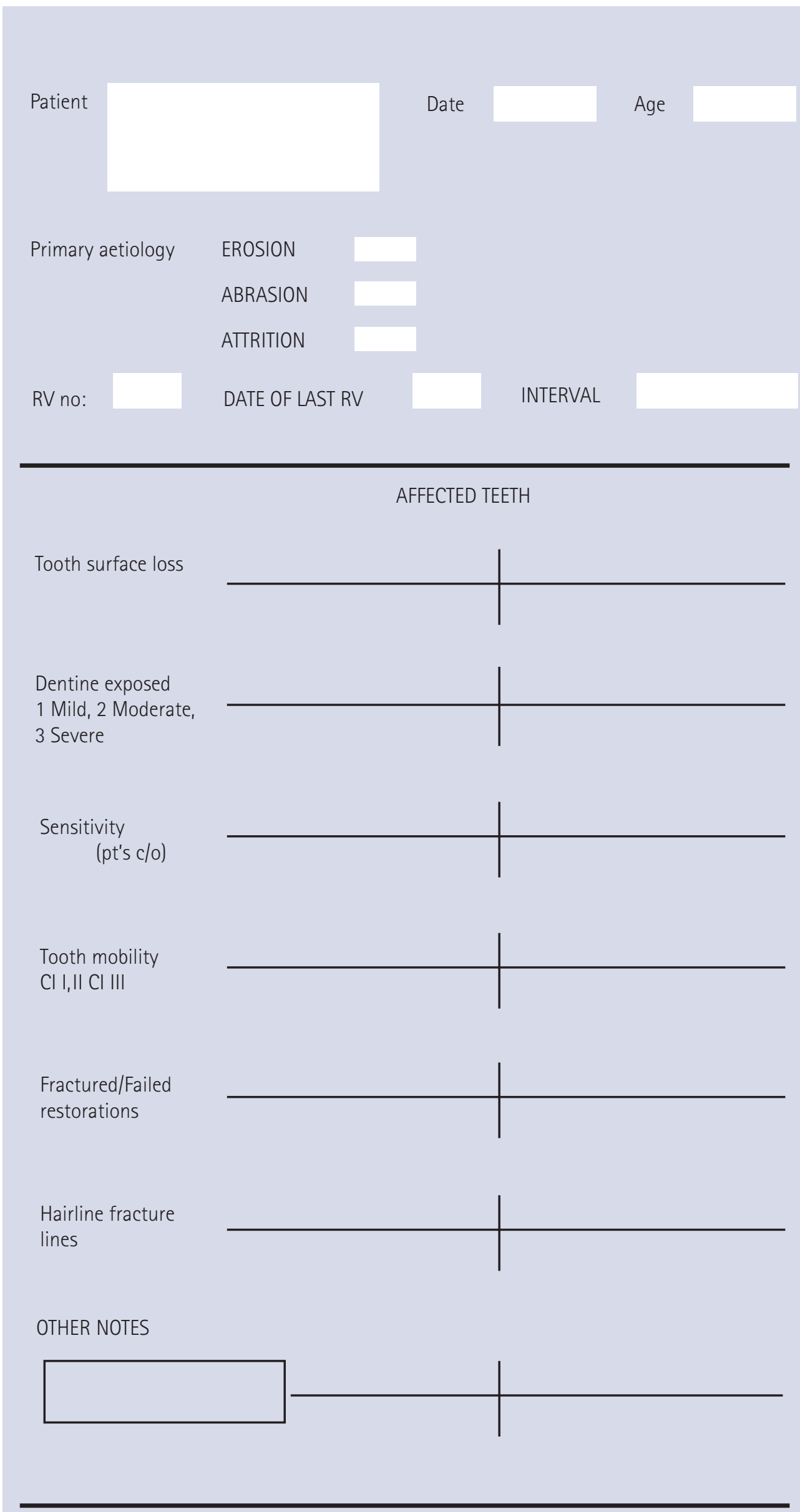

\section{AFFECTED TEETH}

ooth surface loss

Dentine exposed

1 Mild, 2 Moderate

3 Severe

Fig. 6a Tooth surface loss monitoring 
Patient

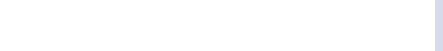

Date

OTHER SIGNS

Active bruxism?

Tongue scalloping

Cheek ridging

T.M.J. exam

Noise

Range of motion

Tenderness to palpitation

Muscle tenderness

RECORDS TAKEN

Photographs Impressions

Measurements from A.D.J.

ASSESSMENT at this time

Non-progressive

Maybe progressive [not marked and no symptoms]

Progressive [obvious]

Progressive [obvious and symptomatic]

TREATMENT at this time

Page 2 of 2 
What are the effects of tooth surface loss? This question gives rise to Box 4 of the examination sheet (Fig. 4).

\section{Has dento-alveolar compensation taken place?}

The effects of tooth surface loss: Physiological tooth surface loss is normal and results in a reduction in both vertical tooth height and horizontal tooth width.

In physiological tooth surface loss, vertical dimension is maintained by alveolar bone remodelling resulting in an elongation of the (dento-)alveolar process, similarly proximal wear is compensated by a constant forward pressure maintaining tooth to tooth contact.

If pathological vertical tooth surface loss has occurred then there is the possibility that a compensatory growth ('dento-alveolar compensation') may have occurred to some degree. This is an important consideration.

\section{Dento-alveolar compensation}

If tooth surface loss affecting the occlusal surfaces of the teeth has occurred, then one might expect to see a reduction in occlusal face height (vertical dimension of occlusion or VDO); or, expressed in a different way, an increase in the freeway space (FWS) could be anticipated. This may be further complicated by forward posturing of the mandible. It is often observed, however, that despite overall tooth surface loss, the freeway space and the resting facial height appear to remain unaltered primarily because of dento-alveolar compensation.

This is important with respect to patient assessment. If restoration of worn teeth is being planned then the extent of dento-alveolar compensation would appear to determine the dentist's strategy; defining the need to carry out measures such as crown lengthening, to ensure the same VDO and FWS .

Nevertheless, the fundamental question is:

'Does it matter if the patient's VDO is increased during the restoration of the tooth surface loss (ie the FWS is reduced)?'

The answer is different for each patient. No occlusion can be said to be 'wrong' rather it is the case that in certain patients, at particular times in their lives, some occlusal patterns will not be tolerated. An occlusion can only be judged by the reaction of the tissues surrounding it, so it is with the issue of an increase in VDO.

In the case of a patient presenting with some dento-alveolar compensation, the clinician should assess whether or not that patient can tolerate an increased VDO (reduced FWS) by the construction of a stabilisation splint and/or provisional restorations.
The incidence of dento-alveolar compensation

It has been observed that in the normal adult dentition, the FWS remains constant and even in those patients who exhibit significant tooth surface loss the VDO is unaffected in 80\% and a normal FWS of $3 \mathrm{~mm}$ is exhibited. If treatment of a patient within this group is necessary then crown lengthening procedures may be indicated. This will enable adequate reduction of the teeth at crown preparation so allowing the same VDO to be maintained. Alternatively restoration of the patient's dentition may be provided at an increased VDO (reduced FWS). Some may argue that any increase in FWS should be proportionate to the degree of attrition.

Does the patient want/need treatment?

This question gives rise to Box 5 of the examination sheet (Fig. 4).

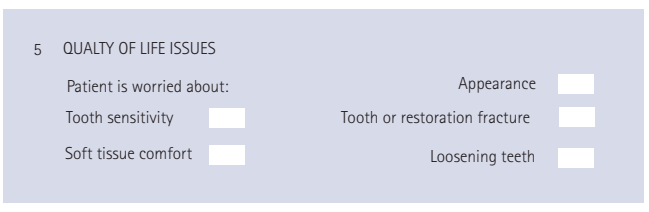

Patient's wants and needs

It is essential as in all areas of clinical practice to carefully consider the patient's anxieties and desires in addition to the clinical features before advice is given.

\section{Monitoring}

Monitoring involves taking a series of repeated examinations and certain measurements over a period of time in order to assess whether a condition is progressive. Monitoring is essential in the management of tooth surface loss.

In the literature several methods of assessing tooth wear have been described including:

- General assessment of extracted teeth,

- Chemical analysis

- Physical methods (polarised light/indentation techniques/profilometry) scanning electron microscopic analysis

- Digital image analysis.

These are research tools and are not applicable to clinical practice. Therefore, a monitoring protocol to assess tooth surface loss is presented (Fig. 6a,b). It is easy to use and by this method it is easy to record the progression of tooth surface loss.

Monitoring is, of course, only an option when baseline measurements have been taken. This emphasises the need for the dentist to examine and record tooth surface loss. To facilitate this a protocol for the initial examination of a patient with TSL has been presented (Fig. 4).

\section{Treatment}

The key question to be answered is:

'Does this patient need treatment?'
Tooth Surface Loss

Has dento-alveolar compensation taken place? 
There are no hard and fast rules and the need for treatment should be established after considering:

- The degree of wear relative to the age of the patient

- The aetiology

- The symptoms

- The patient's wishes.

\section{Treatment may be either passive or active}

\section{Passive treatment}

Monitoring (Figs 6a and 6b)

This has already been discussed under the 'Examination section' and it represents a review process. Monitoring is the only way in which TSL can be assessed as being active or static. It is, therefore, the case that in most situations a period of monitoring should be carried out prior to considering active treatment.

\section{Prevention}

This is 'treatment' of future tooth surface loss. If the extent of existing tooth surface loss is considered to be acceptable, the appropriate treatment is clearly to try to prevent further TSL, which could render the patient needing restorative treatment. The form of the preventive treatment will be dependent on the aetiology of the TSL, so determining the cause is essential.

For a patient whose tooth surface loss is essentially caused by erosive fluids, advice regarding diet, the use of sugar free chewing gum, and the prescription of a fluoride mouthwash will almost certainly be indicated. It may be also necessary to liaise with the doctor if you suspect the patient suffers from a depressive illness.

If the wear is primarily caused by abrasion then examination and modification of the tooth cleaning habits will be indicated.

If the wear is caused by attrition, then the patient should be advised of any possible bruxist habits. The provision of one of three different sorts of splints could be considered. A soft bite guard can help in breaking a bruxist habit or simply will protect the teeth during the bruxist habit. A localised occlusal interference splint is designed to break the bruxist habit, and can be worn easily during the day. A stabilisation splint reduces bruxism by providing an ideal occlusion: it also enables the clinician to locate and record centric relation. ${ }^{9}$

\section{Active treatment}

Non-carious loss of tooth tissue may require treatment for one or more of the following reasons:

- Sensitivity

- Aesthetics

- Function

- Space loss in the vertical dimension.
The latter may present a critical problem and both the need for restorative treatment and the complexity of that restorative treatment may depend upon whether or not dentoalveolar compensation has occurred, as previously discussed.

Where do we start?

Careful planning regarding the reconstruction of a worn dentition is essential and, as in any other restorative treatment plan, the first decision to be made is whether or not the restorations will be designed to:

- Harmonise with the existing occlusion ie the conformative approach, or

- Make a change towards an ideal occlusion ie the reorganised approach.

\section{THE CONFORMATIVE APPROACH}

The following criteria should be met if the conformative approach is to be considered:

1 The patient has an ideal occlusion, with centric occlusion occurring in centric relation, and the anterior guidance is at the front of the mouth. (This is not a common finding)

2 The patient does not have an ideal occlusion. However, the teeth to be restored are not deflecting contacts (those contacts which guide the mandible into their centric occlusion).

3 It is not predicted that any other teeth in the mouth will need to be restored because of further tooth surface loss.

4 There is no temporomandibular disorder.

\section{THE REORGANISED APPROACH}

If these criteria do not pertain, then a re-organised approach will need to be considered.

\section{MANAGEMENT OF A PATIENT WITH PATHOLOGICAL TOOTH SURFACE LOSS}

\section{Treat or monitor?}

Whether or not a particular patient exhibiting TSL needs treatment or monitoring can only be determined by a complex series of decisions. An attempt to illustrate this process, relatively simply, is presented as Figure 7.

\section{Options for patient needing treatment}

The alternatives for treatment are equally complex if the decision has been taken that a patient needs treatment.

The process starts with an examination of the jaw relationship in which the patient is presently occluding (does $\mathrm{CO}$ occur in CR?).

From that decision it is possible to provide some structure to the treatment planning depending upon other factors. Figure 8 is an attempt to present this in a logical sequence. 


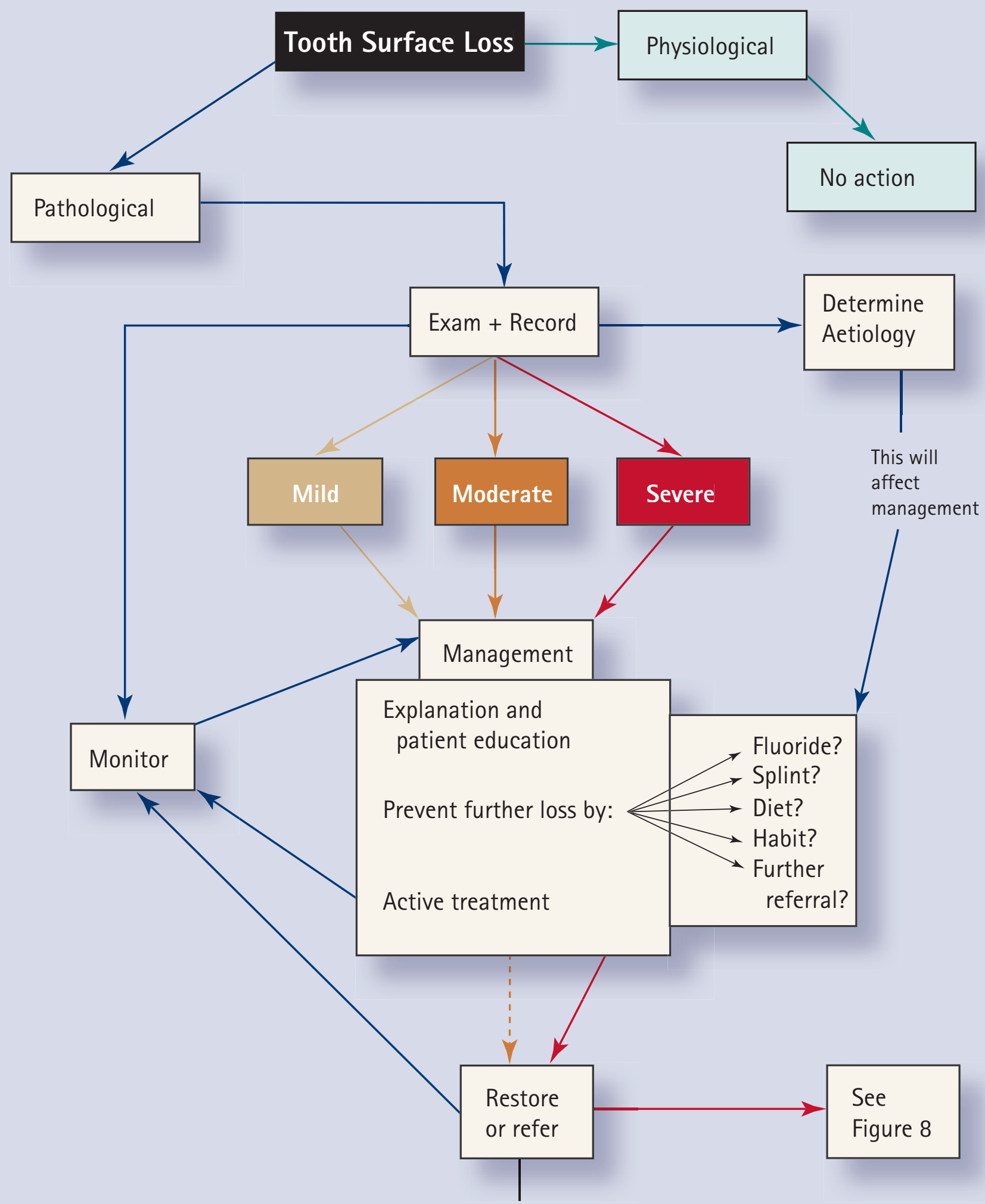

Depends upon:

- Cost

- Time

- Operator experience

- Extent of treatment

- Patient requirement 


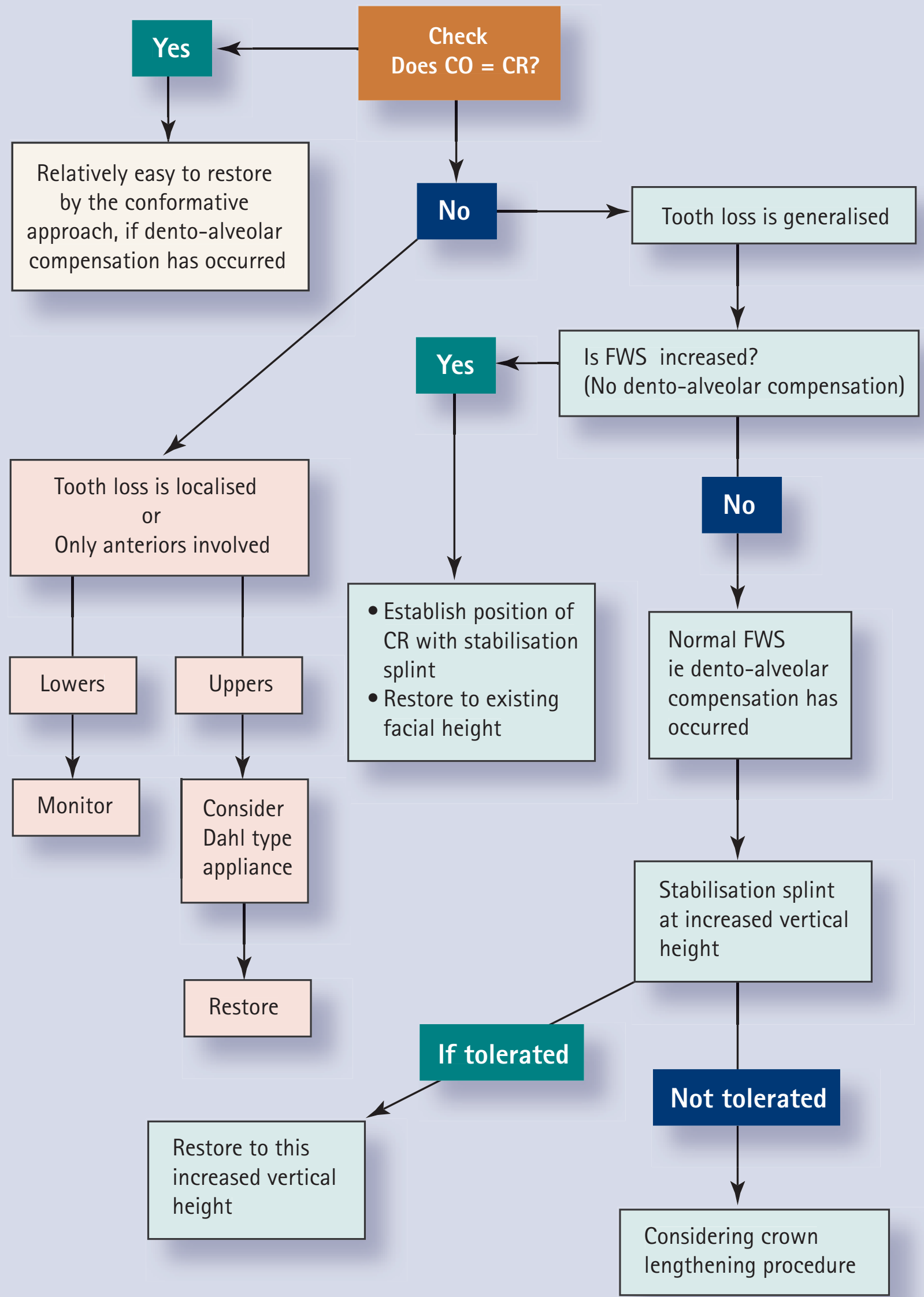




\section{Guidelines of good occlusal practice}

I The examination of the patient involves the teeth, periodontal tissues and articulatory system.

2 There is no such thing as an intrinsically bad occlusal contact, only an intolerable number of times to parafunction on it.

3 The patient's occlusion should be recorded, before any treatment is started.

4 Compare the patient's occlusion against the benchmark of ideal occlusion.

5 A simple, two dimensional means of recording the patient's occlusion before, during and after treatment is an aid to good occlusal practice.

6 The conformative approach is the safest way of ensuring that the occlusion of a restoration does not have potentially harmful consequences.

7 Ensuring that the occlusion conforms (to the patient's pre-treatment state) is a product of examination, design, execution and checking (EDEC)

8 The 'reorganised approach' involves firstly the establishment of a 'more ideal' occlusion in the patient's pretreatment teeth or provisional restorations; and then adhering to that design using the techniques of the 'conformative approach'

9. An 'ideal occlusion' in removable prosthodontics is one which reduces de-stabilising forces

10. The occlusal objective of orthodontic treatment is not clear, but a large discrepancy between centric occlusion and centric relation should not be an outcome of treatment

II. An 'orthodontic' examination of the occlusion should include: the dynamic occlusion; and the jaw relationship in which the patient has centric occlusion

12. The occlusion of periodontally compromised teeth should be designed to reduce the forces to be within the adaptive capabilities of the damaged periodontia

13. Good occlusal practice in children is determined by the needs of the developing occlusion, consequentially 'restoration at all costs' may not be the best policy.

14. Not all tooth surface loss needs treatment, but effective monitoring is essential

15. Dento-alveolar compensation has often occured in patients exibiting marked tooth surface loss.
Flint S, Scully C. Orofacial changes and related disease. Dent Update 1988; 15: 337-342.

2 Milosevic A. Eating disorders and the dentist. BrDent J 1999; 186: 109-113.

3 Braem M, Lambrechts P, Vanherle G. Stress-induced lesions. J Prosthet Dent 1992; 67: 718-722.

4 GrippoJ A. A new classification of hard tissue lesions. J Aesthetic Dent 1988; 3: 14-19.

5 Dawson P E. Evaluation, diagnosis and treatment of occlusal problems. St Louis: CV Mosby Co. 2nd ed. 1989, p457.

6 Franks A S T. Masticatory muscle hypertonicity and temporomandibular joint dysfunction. J Prosthet Dent 1965; 6 1122-1131.

7 Smith B G N, Knight J K. An index for measuring the wear of teeth. BrDent $J$ 1984; 156: 435-438.

8 Kelleher M, Bishop K. Tooth surface loss: an overview. BrDent J 1999; 186: 61-66.

9 Gray R M J, Davies S J, Quayle A A. A clinical approach to temporomandibular disorders: Splint therapy. BrDent J 1994; 177: 135142. 